\title{
Pythagoräische Tripel höherer Ordnung
}

\author{
Von \\ Edmund Hlawka \\ (Vorgelegt in der Sitzung der math.-nat. Klasse am 27. April 2006 \\ durch das w. M. Edmund Hlawka)
}

\section{$\$ 1$.}

Wir wollen das Paar $(A, B)(A, B$ ganze Zahlen, o.B.d.A. $A>B>0)$ als Glied einer Folge

$$
(A(g), B(g))
$$

auffassen, wobei $g$ alle ganzen Zahlen durchläuft.

Wir setzen zunächst

$$
A(0)=A, \quad B(0)=B,
$$

dann definieren wir für $g \geq 0$

$$
\begin{aligned}
& A(g+1)=\left(A^{2}(g)+B^{2}(g)\right)^{1 / 2}=\sqrt{S(g)}, \\
& B(g+1)=(2 A(g) B(g))^{1 / 2}=\sqrt{P(g)}
\end{aligned}
$$

und für $g \leq 0$

$$
\begin{aligned}
& A(g-1)=\frac{1}{2}(\sqrt{S(g)}+\sqrt{D(g)}), \\
& B(g-1)=\frac{1}{2}(\sqrt{S(g)}-\sqrt{D(g)}) .
\end{aligned}
$$

Dabei haben wir

$$
S(g)=A^{2}(g)+B^{2}(g)
$$


und

$$
D(g)=A^{2}(g)-B^{2}(g)
$$

gesetzt.

Ist $g$ nichtnegativ, so erhalten wir die Rekursionsformel

$$
S(g+1)=(A(g)+B(g))^{2}
$$

und für nicht positives $g$

$$
S(g-1)=(A(g))^{2} .
$$

Analog erhalten wir für $g>0$

$$
D(g+1)=(A(g)-B(g))^{2}
$$

und für negatives $g$

$$
D(g-1)=\left(A^{4}(g)+B^{4}(g)\right)^{1 / 2}=\sqrt{S(g) D(g)} .
$$

Weiters ist für $g>0$

$$
P(g+1)=2 \sqrt{P(g) S(g)}
$$

und für negatives $g$

$$
P(g-1)=(B(g))^{2} .
$$

\section{$\$ 2$.}

Wir definieren nun den Winkel $\vartheta(g)$ mit $\vartheta(0)=\vartheta$. Diesen Winkel habe ich in der Arbeit [3] definiert durch

$$
e^{i \pi \vartheta}=\frac{A+i B}{A-i B} .
$$

Er ist nach SCHERRER und HADWIGER irrational. Beschränken wir uns auf den Fall $g \geq 0$, so definieren wir

$$
\begin{aligned}
e^{i \pi \vartheta(g+1)} & =\frac{A(g+1)+i B(g+1)}{A(g+1)-i B(g+1)} \\
& =\frac{(A(g+1)-i B(g+1))^{2}}{A^{2}(g+1)+B^{2}(g+1)} \\
& =\frac{A^{2}(g+1)-B^{2}(g+1)-2 i A(g+1) B(g+1)}{A^{2}(g+1)+B^{2}(g+1)} \\
& =\frac{D(g+1)+i P(g+1)}{S(g+1)} .
\end{aligned}
$$


Weiters definieren wir ( $h$ ganze Zahl)

$$
e^{i \pi h \vartheta}=\frac{A_{h}(g+1)+i B_{h}(g+1)}{A_{h}(g+1)-i B_{h}(g+1)},
$$

und es ist

$$
A_{h}(g+1)+i B_{h}(g+1)=(A(g+1)+i B(g+1))^{4} .
$$

Es ist

$$
D(g+1)=(A(g)-B(g))^{2}
$$

und

$$
P(g+1)=2 \sqrt{P(g) S(g)} .
$$

Es ist $(g \geq 0)$

$$
\cos \pi \vartheta(g+1)=\frac{(A(g)-B(g))^{2}}{(A(g)+B(g))^{2}}=\frac{D(g)}{(A(g)+B(g))^{2}}
$$

und

$$
\begin{aligned}
\sin \pi \vartheta(g+1) & =\frac{2 \sqrt{P(g) S(g)}}{(A(g)+B(g))^{2}} \\
& =\frac{2 \sqrt{2 A B\left(A^{2}(g)+B^{2}(g)\right)}}{A^{2}(g)+B^{2}(g)+2 A(g) B(g)},
\end{aligned}
$$

also ist

$$
\sin \pi \vartheta(g+1)=\frac{2 \sqrt{\frac{2 A B}{A^{2}+B^{2}}}}{1+\frac{2 A B}{A^{2}+B^{2}}}=\frac{2 \sqrt{\sin \vartheta(g)}}{1+\sin \vartheta(g)} \geq \sqrt{\sin \vartheta(g)} .
$$

Daraus folgt mit $A=A(g), B=B(g)$ und $\vartheta=\vartheta(g)$

$$
\sin \pi \vartheta(g+1)=\frac{2}{1+\frac{2 A B}{A^{2}+B^{2}}}=\frac{2 \sqrt{\sin \vartheta}}{1+\sin \vartheta} \geq \sqrt{\sin \vartheta} .
$$

Setzen wir $\sqrt{\sin \vartheta}=y$, so ist

$$
\sin \pi \vartheta(g+1)=\frac{2 y}{1+y^{2}}, \quad \cos \pi \vartheta(g+1)=\frac{1-y^{2}}{1+y^{2}},
$$

also ist

$$
e^{i \pi \vartheta}=\frac{(1-i y)^{2}}{(1+y)^{2}}=\frac{1-i y}{1+i y}
$$


Es ist nun nach (3)

$$
\sin \vartheta(g+1) \geq 2 \sqrt{\sin \vartheta(g)},
$$

daraus folgt durch vollständige Induktion

$$
\sin \vartheta(g+1) \geq(\sin \vartheta(g))^{w(g)}
$$

mit $g \geq 0$, wo $w(g)=1 / 2^{g+1}$ und allgemein

$$
\sin \pi h \vartheta(g+1) \geq(\sin \pi h \vartheta(g))^{w(g)} .
$$

Nun ist $\vartheta(0)=\vartheta_{0}$ irrational und $\sin \pi h \vartheta_{0}$ von der Gestalt

$$
\frac{Z}{\left(A^{2}+B^{2}\right)^{h}},
$$

wo $Z$ eine ganze $Z$ ahl $\neq 0$ ist.

Es wird also

$$
\sin \pi h \vartheta(g+1) \geq\left(\frac{2 A_{n} B_{n}}{A^{2}+B^{2}}\right)^{h w(g)} \geq \frac{1}{\left(A^{2}+B^{2}\right)^{h w(g)}} .
$$

Wir betrachten nun die Weylsche Summe

$$
W_{h}=\frac{1}{N} \sum_{h=1}^{N} e^{2 \pi i h \vartheta(g+1)} .
$$

Es ist nun

$$
\left|W_{h}(g)\right| \leq \frac{1}{N} \frac{2}{|\sin h \vartheta(g+1)|} \leq \frac{1}{N}\left(A^{2}+B^{2}\right)^{h w(g)} .
$$

Wir betrachten nun die Folge $(h \vartheta(g+1))$ modulo 1. Nach dem Satz von ERDÖS-TURAN-KOKSMA ist die Diskrepanz dieser Folge

$$
D_{N} \leq C\left(\frac{1}{M}+\sum_{h=1}^{M} \frac{W_{N}(h)}{h}\right),
$$

wo $M$ noch zu wählen und $C$ eine absolute Konstante ist. Wir erhalten zunächst

$$
D_{N} \leq C\left(\frac{1}{M}+\frac{1}{N}\left(A^{2}+B^{2}\right)^{M w(g)} \log M\right) .
$$

Wir wählen nun $M$ so, dass

$$
M w(g) \lg \left(A^{2}+B^{2}\right)-\log N=-\log \lg N
$$


ist, also

$$
M=\frac{\log N-\log \lg N}{w(g) \lg \left(A^{2}+B^{2}\right)}
$$

wird. Wir erhalten somit

$$
D_{N}(g) \leq \frac{20 C \lg \left(A^{2}+B^{2}\right)}{w(g)} \frac{\log \lg N}{\log N}
$$

Setzen wir alles ein, so erhalten wir

$$
D_{N}(g) \leq C_{1} 2^{g+1} \lg \left(A^{2}+B^{2}\right)\left(\frac{\log \lg N}{\log N}\right) .
$$

\section{Beispiel 1.}

$$
\begin{array}{ll}
A(0)=2, & B(0)=1, \\
A(1)=\sqrt{5}, & B(1)=\sqrt{4}=2, \\
A(2)=3, & \ldots \\
\ldots & B(3)=2 \sqrt{20}, \\
A(4)=49, & B(4)=2 \sqrt{60} .
\end{array}
$$

Allgemein: Wir nehmen eine Primzahl von der Form $p=4 k+1$ und definieren

$$
T(s)=\sum_{x=0}^{p-1}\left(\frac{x}{p}\right)\left(\frac{x^{2}+s}{p}\right),
$$

wo $T(s)$ eine gerade Zahl ist. Es sei nun $s=r$, wenn $r$ quadratischer Rest modulo $p$, also $(r / p)=1$, ist. Wir schreiben $s=n$, wenn $n$ Nichtrest modulo $p$, also $(n / p)=-1$, ist. $p$ besitzt dann die Darstellung

$$
p=\left(\frac{1}{2} T(r)\right)^{2}+\left(\frac{1}{2} T(n)\right)^{2}
$$

und lässt sich - wie schon von FERMAT her bekannt - als Summe zweier Quadrate darstellen. Die Darstellung ist abgesehen von Vorzeichen und Vertauschungen eindeutig. Die obige Darstellung stammt von JABOBSTHAL. Man kann also für $A$ die Darstellung $\frac{1}{2} T(r)$ und für $B$ die Darstellung $\frac{1}{2} T(n)$ nehmen.

\section{$\S 3$.}

Wir setzen jetzt (wieder $g \geq 0$ )

$$
k^{\prime}(g+1)=\left(\frac{B(g+1)}{A(g+1)}\right)^{2}=\frac{P(g)}{S(g)} .
$$


Es ist

$$
\sqrt{k^{\prime}(g+1)}=\left(\frac{B(g+1)}{A(g+1)}\right)^{2}=\sqrt{\frac{P(g)}{S(g)}}
$$

(wir nehmen also das positive Vorzeichen der Quadratwurzel).

Wir definieren weiters, wobei wir $A(g+1)$ bzw. $B(g+1)$ kurz mit $A$ bzw. $B$ bezeichnen,

$$
k(g+1)=\frac{1-k^{\prime}(g+1)}{1+k^{\prime}(g+1)}=\frac{A^{2}-B^{2}}{A^{2}+B^{2}}=\frac{D(g+1)}{S(g+1)} .
$$

Wir erhalten also

$$
k^{2}(g+1)+k^{\prime 2}(g+1)=1 .
$$

Es wird

$$
k^{\prime}(g+1)=\frac{2 \frac{B(g)}{A(g)}}{1+\frac{B^{2}(g)}{A^{2}(g)}}=\frac{2 \sqrt{k^{\prime}(g)}}{1+k(g)}
$$

und es wird

$$
1+k(g+1)=\frac{2 A^{2}(g)}{S(g)}=1+\frac{1-k^{\prime}}{1+k^{\prime}}=\frac{2}{1+k^{\prime}(g+1)} .
$$

Wir definieren nun die Größen $a(g)$ und $b(g)$, wobei

$$
a(g)=S+P, \quad b(g)=S-P,
$$

die wieder positiv sind, und wenden den so genannten arithmetischgeometrischen Algorithmus (kurz AG) an. Wir setzen

$$
a_{1}(g)=\frac{1}{2}(a(g)+b(g)), \quad b_{1}(g)=\sqrt{a(g) b(g)} .
$$

Im Fall (6) ist

$$
a_{1}(g)=S(g), \quad b_{1}(g)=\sqrt{S^{2}-P^{2}}=D .
$$

Wir setzen den Prozess weiter fort,

$$
a_{2}(g)=\frac{a_{1}(g)+b_{1}(g)}{2}, \quad b_{2}(g)=\sqrt{a_{1}(g) b_{1}(g)}
$$

und so weiter.

Im zweiten Fall wenden wir AG auf das Paar

$$
a^{\prime}(g)=S(g)+D(g), \quad b^{\prime}(g)=S(g)-D(g)
$$


an. Es wird

$$
a_{1}^{\prime}(g)=S(g), \quad b_{1}^{\prime}(g)=\sqrt{S^{2}(g)-D^{2}(g)}=P .
$$

GAUSS hat nun Folgendes gezeigt: Besteht das Ausgangspaar $(a, b)$ aus positiven Zahlen kleiner als Eins und konstruieren wir die zugehörige AG-Folge $\left(a_{j}, b_{j}\right)$, so sind die beiden Folgen $\left(a_{j}\right)$ und $\left(b_{j}\right)$ beide konvergent und konvergieren zum gleichen Grenzwert $M(a, b)$, den GAUSS den arithmetisch-geometrischen Grenzwert nennt. Es gilt weiter

$$
\left|a_{j}-b_{j}\right| \leq \frac{|a-b|}{2^{j}} .
$$

Der Grenzwert konvergiert also sehr rasch. Die Folge $a_{j}$ ist monoton wachsend, die Folge $b_{j}$ monoton abnehmend.

Bei der Folge (6) gilt

$$
\frac{\pi}{2} \frac{1}{M(a, b)}=\int_{0}^{\pi / 2} \frac{d \varphi}{\sqrt{a^{2} \cos ^{2} \varphi+b^{2} \sin ^{2} \varphi}},
$$

wo $a=S$ und $b=D$ ist.

Im Fall $\left(6^{\prime}\right)$ gilt

$$
\frac{\pi}{2} \frac{1}{M\left(a^{\prime}, b^{\prime}\right)}=\int_{0}^{\pi / 2} \frac{d \varphi}{\sqrt{a^{\prime 2} \cos ^{2} \varphi+b^{\prime 2} \sin ^{2} \varphi}}
$$

mit $a^{\prime}=S$ und $b^{\prime}=P$.

Heben wir jetzt $s$ in $k$ bzw. $k^{\prime}$ heraus, ersetzen also die Folge $(a(g), b(g))$ durch die Folge $(1, k(g))$ bzw. $\left(1, k^{\prime}(g)\right)$, so erhalten wir

$$
\frac{\pi}{2} \frac{1}{M(1, k)}=\int_{0}^{\pi / 2} \frac{d \varphi}{\sqrt{1-k^{2} \sin ^{2} \varphi}}=K
$$

und

$$
\frac{\pi}{2} \frac{1}{M\left(1, k^{\prime}\right)}=\int_{0}^{\pi / 2} \frac{d \varphi}{\sqrt{1-k^{\prime 2} \sin ^{2} \varphi}}=K^{\prime} .
$$

$K$ und $K^{\prime}$ sind die so genannten vollständigen Integrale erster Gattung in der Legendreschen Normalform, also Funktionen von $k$ bzw. $k^{\prime}$. ein,

Wir setzen weiter $q=e^{\pi i \tau}, \tau=i K^{\prime} / K$ und führen die $\vartheta$-Funktion

$$
\vartheta_{3}(v)=\sum q^{n^{2}} e^{2 \pi i v}
$$


wo $\vartheta_{2}(v)=\vartheta_{1}$ und $\vartheta_{1}(v)=\vartheta_{1}$ und die Nullwerte $\vartheta_{j}(0)$ sind. Weiters setzen wir $u=\pi \vartheta_{3}^{2}(v)$,

$$
\begin{aligned}
\operatorname{sn} u & =\frac{1}{\sqrt{k}} \frac{\vartheta_{1}(v)}{\vartheta_{0}(v)}, \\
\operatorname{cn} u & =\sqrt{\frac{k^{\prime}}{k} \frac{\vartheta_{2}(v)}{\vartheta_{0}(v)},} \\
\operatorname{dn} u & =\sqrt{k^{\prime}} \frac{\vartheta_{3}(v)}{\vartheta_{0}(v)} .
\end{aligned}
$$

Es ist

$$
k=\left(\frac{\vartheta_{2}}{\vartheta_{3}}\right)^{2}, \quad k^{\prime}=\left(\frac{\vartheta_{0}}{\vartheta_{3}}\right)^{2}
$$

(vgl. [4], S. 53).

\section{Literatur}

[1] HLAWKa, E. (1999) Über einige geometrische Anwendungen im Zusammenhang mit Pythagoräischen Tripeln und Gleichverteilung. Aequationes Math. 58: 163-175

[2] Hlawka, E. (1999) Pythagorean Tripels. In: Bambah, R. P., DumiR, V. C., HANs-GILl, R. J. (eds.) Number Theory, pp. 141-155. Hindustan Book Agency, New Delhi

[3] Hlawka, E. (Preprint) Über einige geometrische Anwendungen der Pythagoräischen Tripel, Teil III

[4] Krause, M. (1912) Theorie der elliptischen Funktionen (Mathematisch-physikalische Schriften für Ingenieure und Studierende, 13). B. G. Teubner, Stuttgart

Anschrift des Verfassers: Prof. Dr. Dr. h.c. Edmund Hlawka, Margarethenstraße 27/II/9, 1040 Wien, Austria. 